\title{
Clave dicotómica para la identificación de Apendicularias en el PacíficoTropical Oriental y Mares Interamericanos, con un listado de especies para Costa Rica
}

\author{
Marco Corrales-Ugalde ${ }^{1,2}$, Iván Castellanos-Osorio ${ }^{3}$ \& Álvaro Moráles-Ramírez ${ }^{1,4}$ \\ 1. Centro de Investigación en Ciencias del Mar y Limnología (CIMAR), Universidad de Costa Rica, San Pedro, 11501- \\ 2060 San José, Costa Rica; mcugalde88@gmail.com, alvaro.morales@ucr.ac.cr \\ 2. Oregon Institute of Marine Biology, 5289 University of Oregon, Eugene, OR 97403 USA; mcugalde88@gmail.com \\ 3. El Colegio de la Frontera Sur (ECOSUR) Unidad Chetumal, Av. Centenario km 5.5, A.P. 424, 77000, Chetumal, Quintana \\ Roo, México; ivancast@ecosur.mx \\ 4. Escuela de Biología, Universidad de Costa Rica, San Pedro, 11501-2060 San José, Costa Rica; \\ alvaro.morales@ucr.ac.cr
}

Recibido 16-I-2017. Corregido 28-VI-2017. Aceptado 03-I-2018.

\begin{abstract}
Dichotomous key for the identification of Apendicularians in the Eastern Tropical Pacific and Inter American Seas, with a species list for Costa Rica. Although appendicularians are relevant primary consumers and are part of every zooplankton community, there is a lack of information on the species present in different ocean regions. Thus, regional identification guides are useful to develop datasets with high taxonomic resolution. Appendicularian species were identified in 33 epipelagic samples of zooplankton collected in several locations of Costarican waters. Eighteen appendicularian species were identified, of which 15 were found in the Pacific and only three in the Caribbean. Seven species are new records for Costa Rican Pacific waters (Appendicularia sicula, Fritillaria charybdae, F. cf. pacifica, F. tenella, F. pellucida f. omani, Oikoipleura fusiformis f. cornutogastra and Pelagopleura verticalis). Data for each species distribution in Costa Rica is presented together with a key for the identification of appendicularian species recorded in the Inter-American seas and the Eastern Tropical Pacific. Rev. Biol. Trop. 66(Suppl. 1): S108-S122. Epub 2018 April 01.
\end{abstract}

Key words: Pelagic tunicates, Costa Rica, identification key, Caribbean Sea, zooplankton, taxonomy, species list.

Las apendicularias son tunicados pelágicos que han sido objeto de numerosos estudios ecológicos por su relevancia como consumidores primarios (Alldredge, 1981; Bedo, Acuña, Robins, \& Harris, 1993; López-Urrutia, Irigoien, Acuña, \& Harris, 2003). Estos organismos son suspensívoros, y secretan una estructura externa de mucopolisacáridos conocida como "casa", la cual puede seleccionar y retener un amplio ámbito de tamaños de partículas desde 0,2 $\mu \mathrm{m}$ hasta $6 \mu \mathrm{m}$ (Flood \& Deibel, 1998; Madin \& Deibel, 1998). Las casas de las apendicularias retienen casi el $30 \%$ del material que pasa a través de los filtros (Flood \& Deibel, 1998). Este material retenido que se hunde una vez descartada la casa es una fuente importante de carbono para otros organismos como microcrustáceos, ciliados y bacterias (Alldredge, 1976; Davoll \& Youngbluth, 1990). Las larvas de peces y adultos se alimentan tanto de sus casas como de los organismos (Tokioka \& Suárez-Caabro, 1956; Alldredge, 1976; Alldredge \& Madin, 1982).

Las apendicularias también han sido utilizadas como indicadores hidroclimáticos, ya que la composición de especies cambia según las distintas masas de agua (Fenaux \& Dallot, 1980). Para utilizarse como indicadores, una línea base de la distribución de distintas especies es necesaria para entender como las 
poblaciones de estos organismos se distribuyen en relación a variaciones oceanográficas y asi predecir como los ecosistemas marinos pueden ser alterados debido al cambio climático (Stachowicz, Terwin, Whitlatch, \& Osman, 2002; (Richardson, 2008). Sin embargo, en ciertas regiones biogeográficas como el Pacífico Tropical Oriental y el Mar Caribe se cuenta con muy pocos registros y guías de identificación de estos organismos (Costello et al., 2010). Los objetivos de este estudio son (1) determinar las especies de apendicularias presentes en los mares costarricences y (2) presentar una clave dicotómica para las especies reportadas en las regiones epipelágicas del Pacífico Este Tropical (PET) y los Mares Interamericanos (MI).

\section{MATERIALES Y MÉTODOS}

Recolección de las muestras: Los especímenes se recolectaron mediante arrastres con redes cónicas de plancton con aperturas de poro de 100, 200 y $500 \mu \mathrm{m}$. Los sitios de muestreo se detallan en la figura 1. La forma en la cual estos arrastres fuero realizados varía según el sitio y se resume en el cuadro 1. Las muestras se fijaron y preservaron en una disolución de formaldehido en agua de mar al $4 \% \mathrm{v} / \mathrm{v}$. Posteriormente se analizaron en el laboratorio de Zooplancton del Centro de Investigación en Ciencias del Mar y Limnología, de la Universidad de Costa Rica (CIMAR-UCR). Se utilizó un estereoscopio marca Nikon modelo SMZ 660, para observar las características taxonómicas importantes para la determinación de las especies, tales como forma general del tronco, presencia de glándulas prebucales, presencia y cantidad de células subcordales y anficordales. La identificación y descripción de los organismos se realizó de acuerdo a Fenaux (1998b). Las apendicularias identificadas se depositaron en la colección de Cordados del Museo

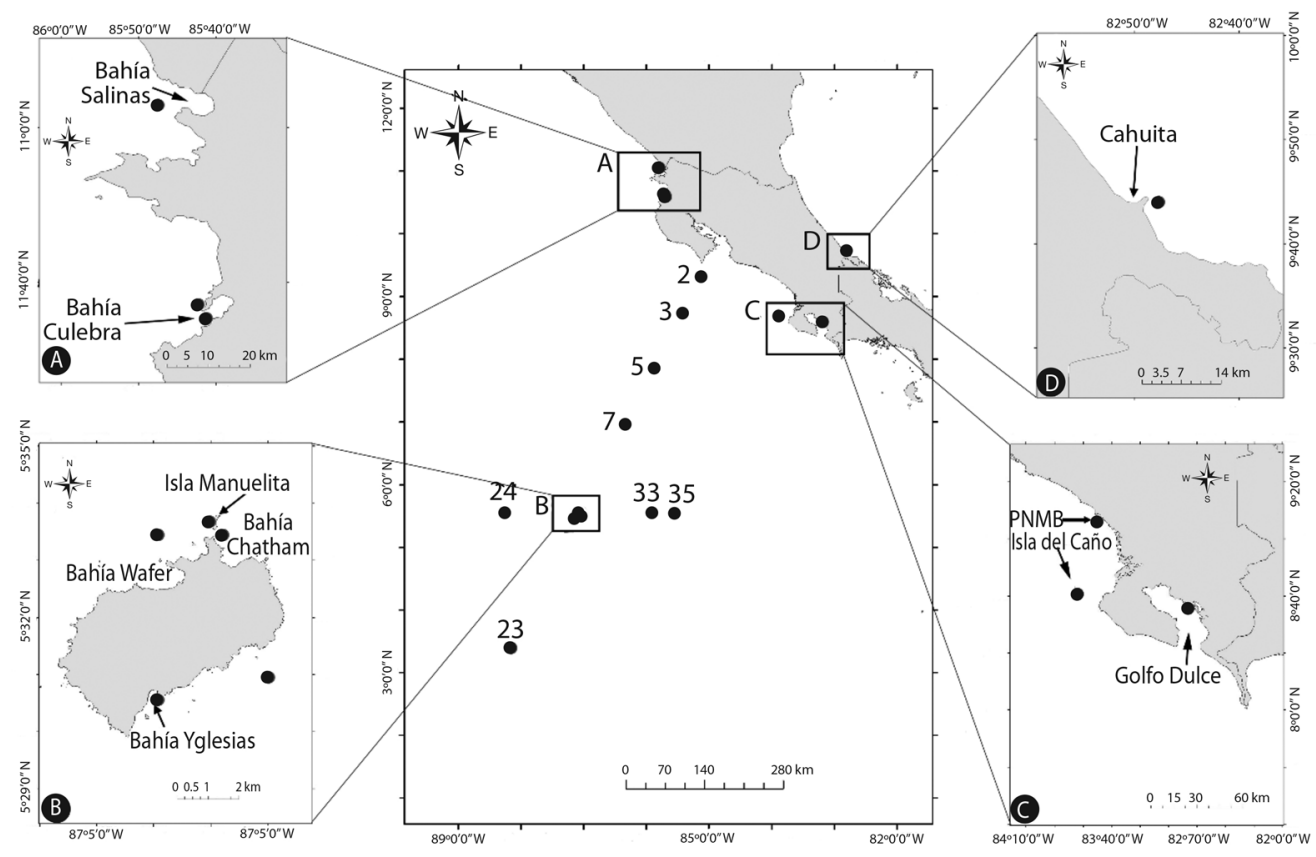

Fig. 1. Sitios de recolecta de las apendicularias en el Océano Pacífico y mar Caribe de Costa Rica. Las ampliaciones del mapa corresponden a las regiones de: A: Pacífico Norte; B: Isla del Coco; C: Pacífico Sur; D: Mar Caribe Sur.

Fig. 1. Sites of sampling of appendicularia in the Pacific Ocean and Caribbean Sea of Costa Rica. The map enlargements correspond to the regions of: A: North Pacific; B: Coco Island; C: South Pacific; D: South Caribbean Sea. 


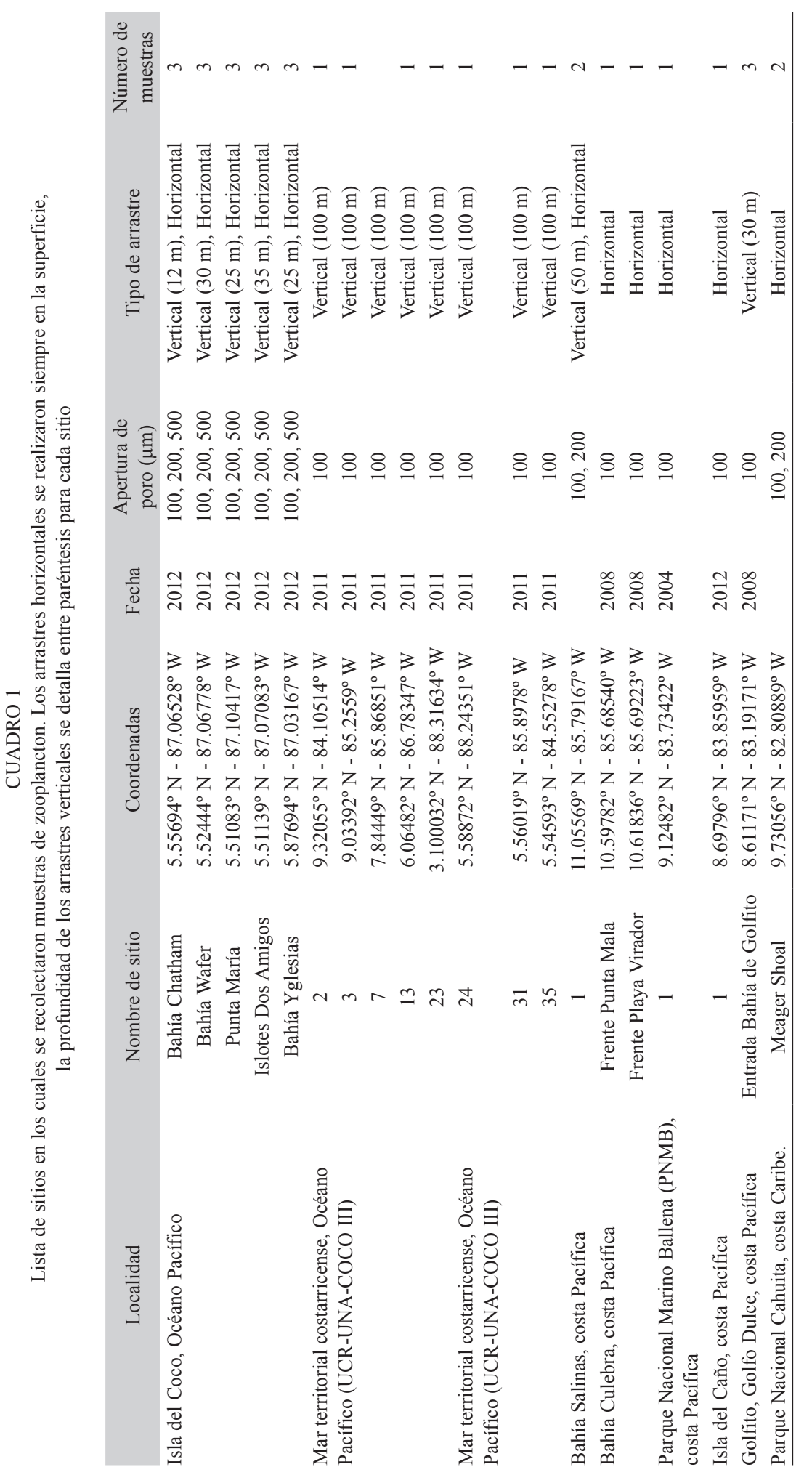


de Zoología de la Universidad de Costa Rica (código MZUCR). Con base en las descripciones morfológicas, se desarrolló una clave dicotómica para la identificación de las especies encontradas en el presente estudio y en otros estudios realizados en las regiones del Pacífico Oriental Tropical y los Mares Interamericanos.

\section{RESULTADOS}

A partir del análisis taxonómico de las apendicularias recolectadas en el Océano Pacífico y mar Caribe de Costa Rica, se determinó un total de 18 especies, pertenecientes a dos familias y cinco géneros. De las especies registradas siete son nuevos registros para el Pacífico Costarricense. En el mar Caribe únicamente se encontraron tres especies (Oikopleura dioica, Oikopleura longicauda y Fritillaria haplostoma). El arreglo sistemático presentado a continuación se basa en Fenaux (1993, 1998a).

\section{Clase Appendicularia}

(Chamisso \& Eysenhardt, 1821)

Orden Copelata Haeckel, 1866

Familia Fritillariidae Lohmann, 1915

Género Appendicularia Fol, 1874

\section{Appendicularia sicula}

Fol, 1874 (Fig. 2A, B)

Número de catálogo: MZUCR 67-01, MZUCR 68-01

Número de especímenes observados: 3

Distribución en Costa Rica: Presente en Bahía Culebra, y las estaciones 2 y 24 de la expedición UCR-UNA-COCOS III. Primer registro para el Pacífico Tropical Oriental.

Género Fritillaria Fol, 1872

\section{Fritillaria charybdae}

Lohmann en Lohmann \& Buckmann, 1926 (Fig. 2C, D)

Número de catálogo: MZUCR 66-07 Número de especímenes observados: 2
Distribución en Costa Rica: Dos individuos recolectados en Bahía Salinas, en Febrero del 2012. Este es el primer registro de la especie para el Pacífico Tropical Oriental

\section{Fritillaria formica f. digitata}

Lohmann en Lohmann \& Buckmann, 1926 (Fig. 2E) 69-05

Número de catálogo: MZUCR 67-02,

Número de especímenes observados: 9

Distribución en Costa Rica: Ampliamente distribuida en el Pacífico, donde Castellanos, Hernández, Morales-Ramírez, \& CorralesUgalde (2012), la registraron en la Isla del Coco. Se encontró en Bahía Salinas, Bahía Culebra, Parque Nacional Marino Ballena, y en la expedición Oceanográfica UCR-UNACOCO III. También fue encontrada en el Parque Nacional Cahuita, donde ha sido reportada previamente (Carrillo, este volumen).

\section{Fritillaria formica f. tuberculata}

Lohmann en Lohmann \& Buckmann, 1926 (Fig. 2F, 3A)

Número de catálogo: MZUCR 68-03

Número de especímenes observados: 5

Distribución en Costa Rica: Presenta la misma distribución que $F$. formica f. digitata.

\section{Fritillaria haplostoma}

Fol, 1872 (Fig. 3B, C)

Número de catálogo: MZUCR 67-03, MZUCR 68-02

Número de especímenes observados: 16

Distribución en Costa Rica: Ampliamente distribuida en el Pacífico, donde Castellanos et al. (2012), la registraron en la Isla del Coco. Se encontró en Bahía Salinas, Bahía Culebra, Parque Nacional Marino Ballena, y en la expedición Oceanográfica UCR-UNA-COCO III. También fue encontrada en el Parque Nacional Cahuita, donde ha sido reportada previamente (Carrillo, este volumen). 

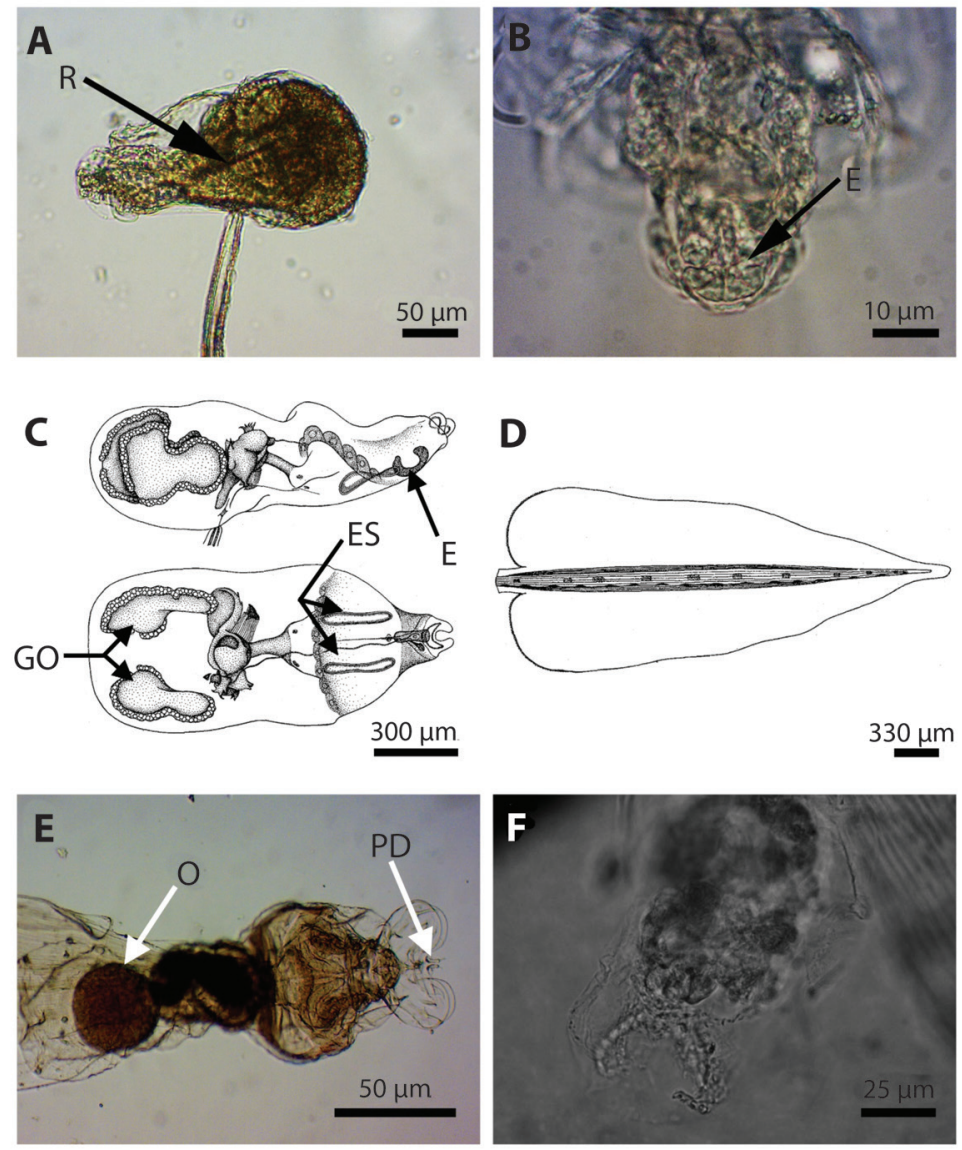

Fig. 2. Appendicularia sícula: A: Tronco, lado izquierdo y B: Detalle del endostilo triangular; Fritillaria charybdae: C: Tronco, vista lateral y dorsal y D: Cola, ambas modificadas de Tokioka (1951); Fritillaria formica: E: Tronco de F. formica f. digitata; F: Detalle de los labios de F. formica f. tuberculata. (R: recto, E: endostilo, ES: espiráculos, GO: gónadas, O: ovario, PD: procesos digitiformes).

Fig. 2. Appendicularia sicula: A: Trunk, left side and B: Detail of triangular endostile detail; Fritillaria charybdae: C: Trunk, lateral and dorsal view and D: Tail, both modified from Tokioka (1951); Fritillaria formica: E: Trunk of $F$. formica f. digitate; F: Lips of $F$. formica f. tuberculata. (R: rectum, E: endostile, ES: spiracles, GO: gonads, O: ovary, PD: digitiform processes).

\author{
Fritillaria cf. pacifica \\ Tokioka 1958 (Fig. 3D, E)
}

Número de catálogo: MZUCR 67-16

Número de especímenes observados: 3

Distribución en Costa Rica: Encontrada

en las estaciones 2 y 3 (UCR-UNA-COCO III). Primer registro para el Pacífico Tropical Oriental.

\section{Fritillaria pellucida f. omani}

Fenaux, 1967 (Fig. 3F, 4A)

Número de catálogo: MZUCR 66-06, MZUCR 67-04

Número de especímenes observados: 14

Distribución en Costa Rica: Presente en las estaciones oceanográficas 2 y 24 de la expedición oceanográfica UCR-UNA-COCO 

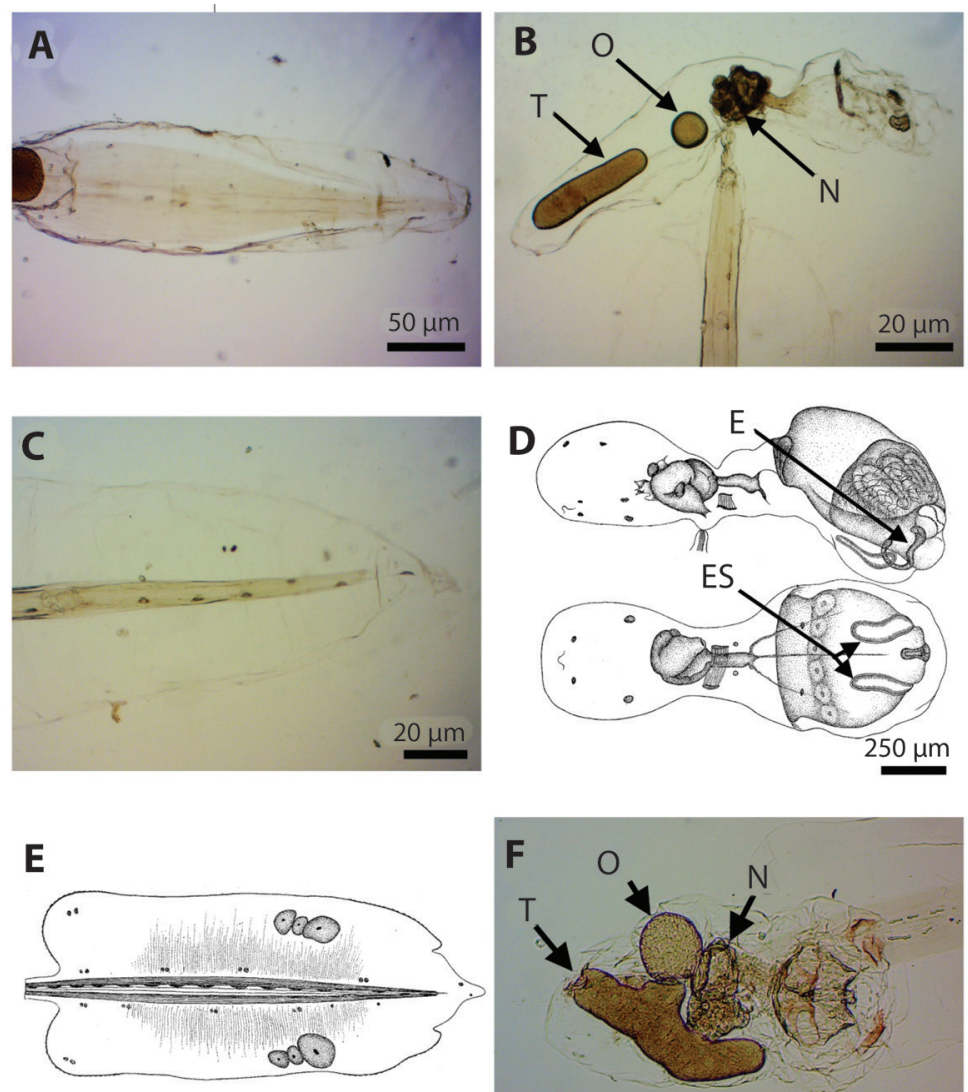

$250 \mu \mathrm{m}$

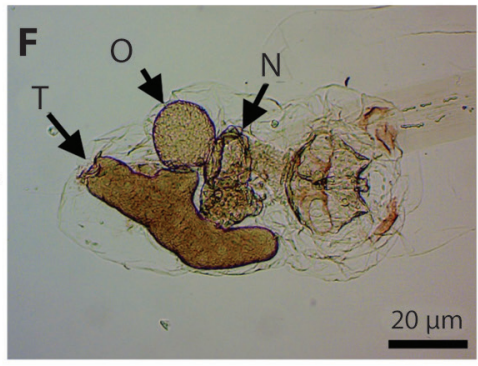

Fig. 3. A: Cola de F. formica f. digitata; Fritillaria halpostoma: B: Tronco, vista lateral y C: Parte distal de la cola; Fritillaria cf. pacifica: D: Tronco, vista lateral y ventral y E: Detalle de la cola, ambas modificadas de Tokioka (1958); Fritillaria pellucida: F: Tronco de F. pellucida omani. (O: ovario, T: testículo, N: núcleo digestivo, E: endostilo, ES: espiráculos).

Fig. 3. A: F. formica f. digitata tail; Fritillaria halpostoma: B: Trunk, lateral view and C: Distal portion of the tail; Fritillaria cf. pacifica: D: Trunk, lateral and ventral view and E: Detail of the tail, both modified from Tokioka (1958); Fritillaria pellucida: F: Trunk of $F$. pellucida omani (O: ovary, T: testes, N: digestive nucleus, E: endostile, ES: spiracles).

III. Este es el primer registro de la especie para Costa Rica

\section{Fritillaria pellucida f. typica}

(Busch, 1851) (Fig. 4A)

\section{Número de catálogo: MZUCR 68-04}

Número de especímenes observados: 6

Distribución en Costa Rica: Presente principalmente en Bahía Culebra y Golfo Dulce, se encontraron pocos individuos en las estaciones oceánicas 2 y 24 de la expedición oceanográfica UCR-UNA-COCO III.

\section{Fritillaria tenella}

Lohmann, 1896 (Fig. 4B)

Número de catálogo: MZUCR 70-01

Número de especímenes observados: 1

Distribución en Costa Rica: Recolectada en Bahía Yglesias, Isla del Coco. Primer registro para el Pacífico Tropical Oriental. 

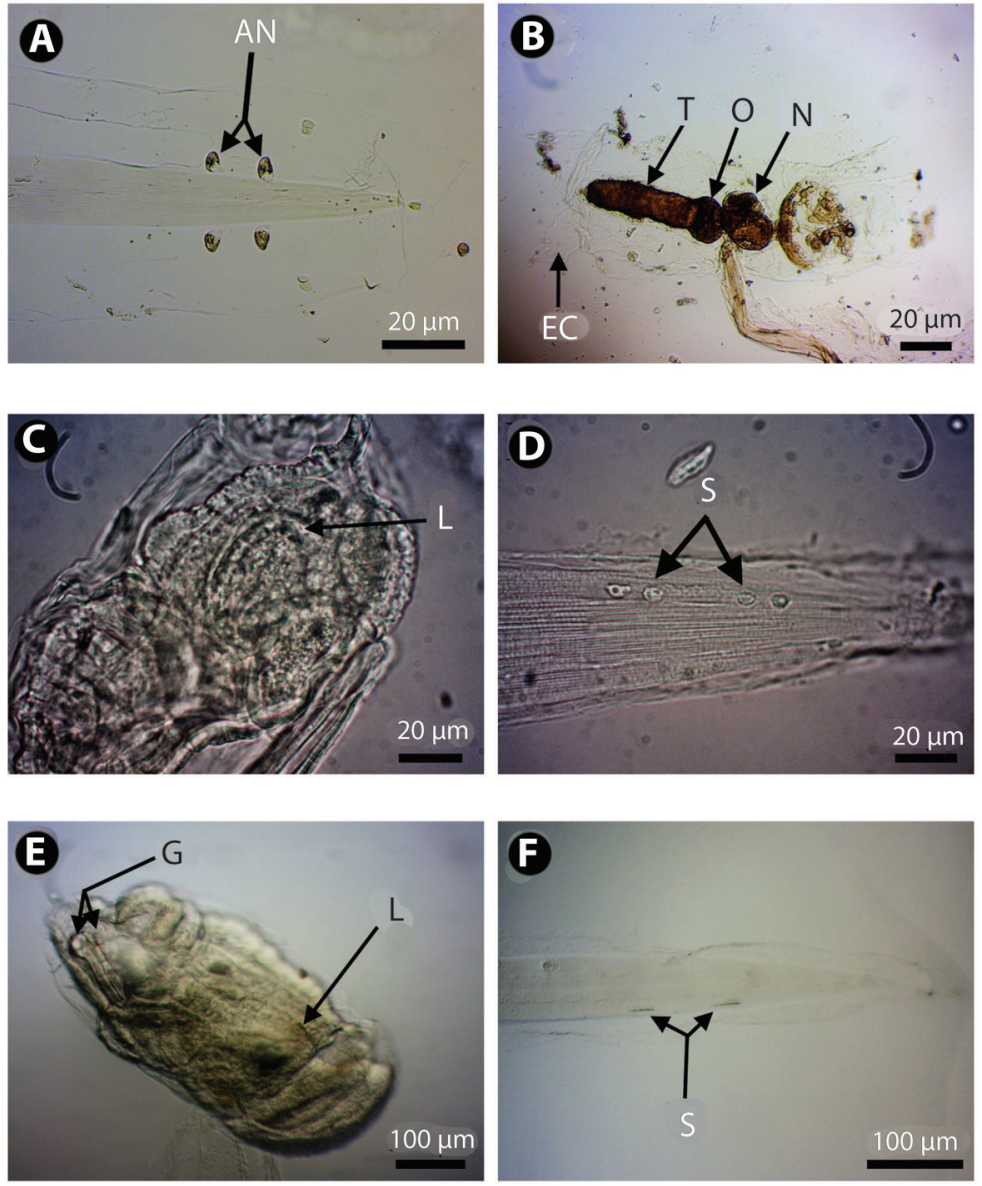

Fig. 4. Fritillaria pellucida: A: Detalle de la cola; Fritillaria tenella: B: Tronco; Oikopleura cophocerca: C: Vista izquierda del tronco y D: Parte distal de la cola; Oikopleura dioica: E: Vista izquierda del tronco y F: Parte distal de la cola. (AN: células anficordales, EC: extensiones de la cutícula, T: testículo, O: ovario, N: núcleo digestivo, L: lóbulo izquierdo del estómago, S: células subcordales, G: glándulas bucales).

Fig. 4. Fritillaria pellucida: A: Detail of the tail; Fritillaria tenella: B: Trunk; Oikopleura cophocerca: C: Trunk and D: Distal part of the tail; Oikopleura dioica: E: Left view of the trunk and F: Distal part of the tail. (AN: anphichordal cells, EC: cuticle extensions, T: testes, O: ovary, N: digestive nucleus, L: left stomach lobe, S: subchordal cells, G: buccal glands).

Familia Oikopleuridae Lohmann, 1915

Género Oikopleura Mertens, 1830

\section{Oikopleura (Vexillaria) cophocerca}

(Gegenbaur, 1855) (Fig. 4C, D)

Número de catálogo: MZUCR 66-01, MZUCR 67-05, MZUCR 68-05, MZUCR 69-02.
Número de especímenes observados: 16

Distribución en Costa Rica: Especie bastante común tanto en ambientes neríticos y oceánicos del Pacífico costarricense. Se ha encontrado en Bahía Salinas, Bahía Culebra, en las estaciones 2, 23 y 24 de la expedición oceanográfica UCR-UNA-COCO III y en varios sitios alrededor de la Isla del Coco (Castellanos et al., 2012). 


\section{Oikopleura (Vexillaria) dioica}

Fol, 1872 (Fig. 4E, F)

Número de catálogo: MZUCR 66-02, MZUCR 66-03, MZUCR 68-06

Número de especímenes observados: 13

Distribución en Costa Rica: Especie común en ambientes neríticosy oceánicos del Pacífico costarricense. Se ha encontrado en Bahía Salinas, Bahía Culebra yen las estaciones 7, 24, 32 y 33 de la expedición oceanográfica UCR-UNA-COCO III, y en varios sitios alrededor de la Isla del Coco (Castellanos et al., 2012).

\section{Oikopleura (Vexillaria) rufescens (Fol, 1872) (Fig. 5A, B)}

Número de catálogo: MZUCR 67-08, MZUCR 68-08, MZUCR 69-04

Número de especímenes observados: 10

Distribución en Costa Rica: Se ha encontrado en Bahía Salinas, Bahía Culebra, Golfo Dulce, en las estaciones 2 y 24 de la
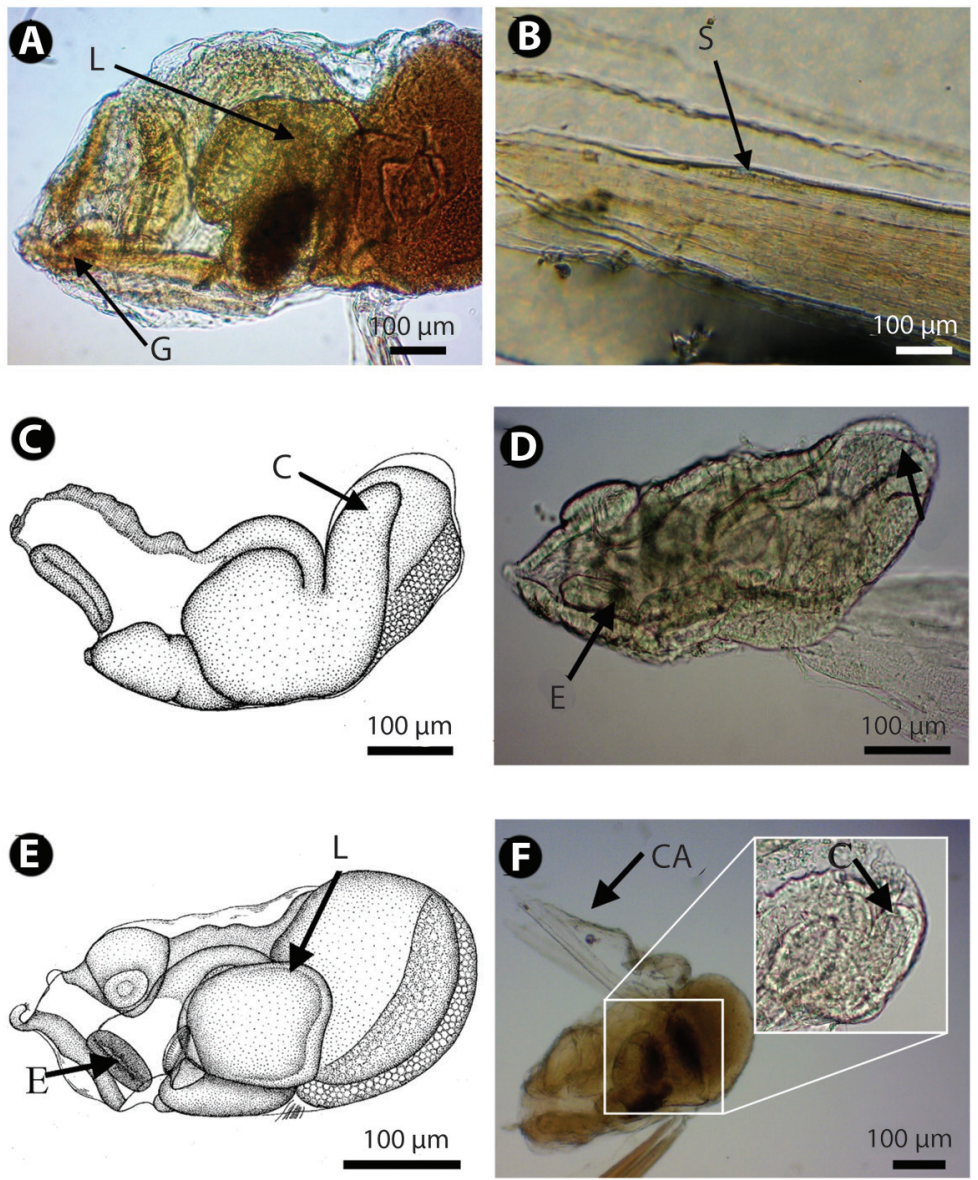

Fig. 5. Oikopleura rufescens: A: Vista izquierda del tronco y B: Parte distal de la cola; Oikopleura fusiformis: C: Tronco de O. fusiformis f. cornutogastra modificado de Tokioka (1951); D: Tronco de O. fusiformis f. typica; Oikopleura gracilis: E: Tronco, modificado de Tokioka (1951); Oikopleura longicauda: F: Tronco. (C: ciego digestivo, E: endostilo, L: lóbulo izquierdo del estómago, CA: capuchón, G: glándulas bucales, S: célula subcordal).

Fig. 5. Oikopleura rufescens: A: Left view of the trunk and B: Distal part of the tail; Oikopleura fusiformis: C: O. fusiformis f. cornutogastra trunk, modified from Tokioka (1951); D: O. fusiformis f. typica trunk; Oikopleura gracilis: E: Trunk, modified from Tokioka (1951); Oikopleura longicauda: F: Trunk. (C: digestive caecum, E: endostile, L: left stomach lobe, CA: hood, G: buccal glands, S: subchordal cell). 
expedición oceanográfica UCR-UNA-COCO III, y en varios sitios alrededor de la Isla del Coco (Castellanos et al., 2012), sin embargo, es mucho más abundante en áreas cercanas a la costa, particularmente en el Pacífico Norte de Costa Rica.

\section{Okiopleura (Coecaria) fusiformis \\ f. cornutogastra Aida, 1907 (Fig. 5C)}

Número de catálogo: MZUCR 66-04, MZUCR 67-17

Número de especímenes observados: 9

Distribución en Costa Rica: Especie común en ambientes neríticos y poco común en ambientes oceánicos del Pacífico costarricense. Se ha encontrado en Bahía Salinas, Bahía Culebra, Golfo Dulce, en las estaciones 2 y 24 de la expedición oceanográfica UCR-UNA-COCO III, y en varios sitios alrededor de la Isla del Coco (Castellanos et al., 2012). Primer registro de esta forma para el Pacífico Costarricense.

\section{Oikopleura (Coecaria) fusiformis}

f. typica Fol, 1872 (5D)

Número de catálogo: MZUCR 67-06

Número de especímenes observados: 12

Distribución en Costa Rica: Misma distribución que $O$. fusiformis f. cornutogastra

Oikopleura (Coecaria) gracilis

Lohmann, 1896 (5E)

Número de catálogo MZUCR: MZUCR 89-06

Número de especímenes observados: 10

Distribución en Costa Rica: Se ha encontrado en Bahía Salinas, Bahía Culebra, Golfo Dulce, en las estaciones 2 y 24 de la expedición oceanográfica UCR-UNA-COCO III, y en varios sitios alrededor de la Isla del Coco (Castellanos et al., 2012)
Oikopleura (Coecaria) longicauda

(Vogt, 1854) (5 F)

Número de catálogo: MZUCR 66-05, MZUCR 67-07, MZUCR 68-07, MZUCR 69-01

Número de especímenes observados: 18

Distribución en Costa Rica: Se ha encontrado en Bahía Salinas, Bahía Culebra, Golfo Dulce, en las estaciones 2, 24 y 35 de la expedición oceanográfica UCR-UNA-COCO III, y en varios sitios alrededor de la Isla del Coco (Castellanos et al., 2012). También fue encontrada en el Parque Nacional Cahuita, donde ha sido reportada previamente (Carrillo, este volumen).

\section{Género Pelagopleura}

Lohmann en Lohmann \& Buckmann, 1926

$$
\begin{gathered}
\text { Pelagopleura cf. verticalis } \\
\text { (Lohmann, 1914) (Fig. 6A, B) (Fig. 6A, B) }
\end{gathered}
$$

Número de catálogo: MZUCR 67-09

Número de especímenes observados: 3

Distribución en Costa Rica: Únicamente ha sido encontrada en la estación 2 de la expedición oceanográfica UCR-UNA-COCO III. Primer registro para el Pacífico Tropical Oriental.

Género Stegosoma Chun, 1888

$$
\begin{gathered}
\text { Stegosoma magnum } \\
\text { (Langerhans, 1880) (Fig. 6C, D) }
\end{gathered}
$$

Número de catálogo: MZUCR 68-09

Número de especímenes observados: 5

Distribución en Costa Rica: Presente principalmente en ambientes oceánicos del Pacífico, se recolectó en la estación 24 de la expedición oceanográfica UCR-UNA-COCO III, y en varios sitios alrededor de la Isla del Coco (Castellanos et al., 2012). Se encontró además un espécimen en el Golfo Dulce. 


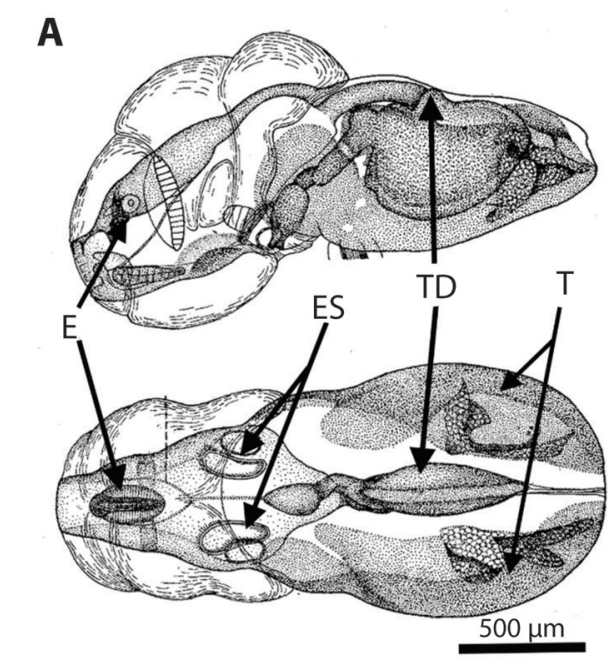

C
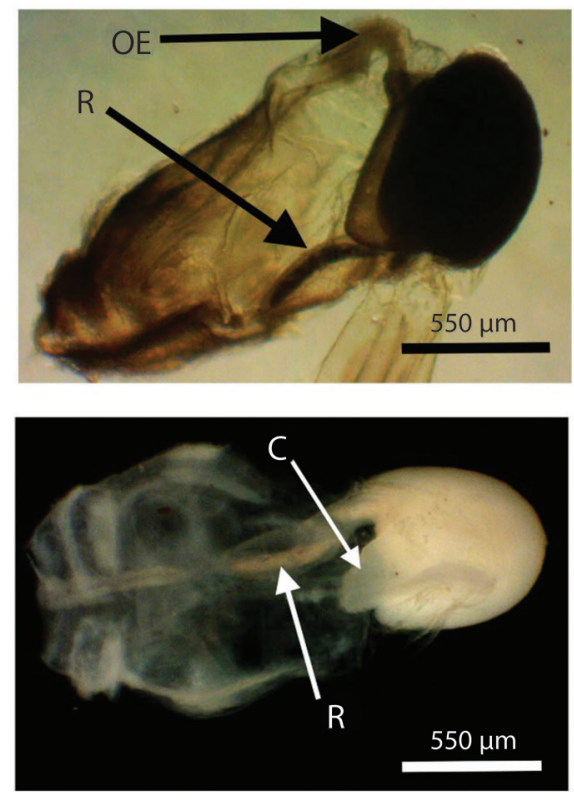

B

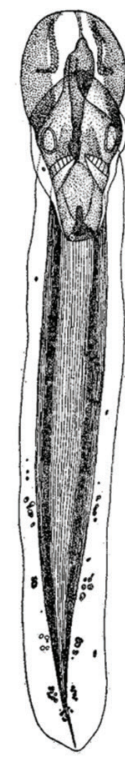

$500 \mu \mathrm{m}$

D

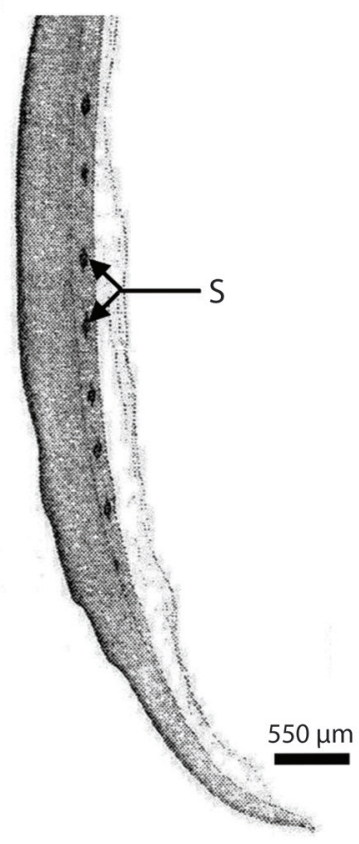

Fig. 6. Pelagopleura verticalis: A: Vista lateral y dorsal del tronco and B: Vista completa del animal, ambas modificadas de Tokioka (1955); Stegosoma magnum: C: Vista lateral y dorsal del tronco; D: Vista lateral de la cola, modificado de Esnal (1996). (E: endostilo, ES: espiráculo, TD: tracto digestivo, T: testículos, ESF: esófago, R: recto, C: ciego digestivo, S: células subcordales).

Fig. 6. Pelagopleura verticalis: A: Lateral and dorsal view of the trunk; B: Whole animal, both modified from Tokioka (1955); Stegosoma magnum: C: Lateral and dorsal view of the trunk; D: Lateral view of the tail, modified from Esnal (1996). (E: endostile, ES: spiracle, TD: digestive tract, T: testes, ESF: oesophagus, R: rectum, C: digestive caecum, S: subchordal cells). 


\section{Clave dicotómica para la determinación de las especies de Appendicularia reportadas para las regiones de los mares interamericanos (MI) y el Pacífico Este Tropical (PET).}

1a. Sin endostilo. Cavidad faríngea con dos bandas ciliadas. Tejido oikoplástico conformado por una sola célula grande .

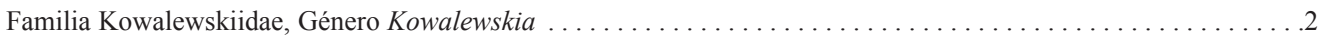

1b. Con endostilo. Cavidad faríngea sin bandas ciliadas. Tejido okioblástico conformado por varias células. . . . . . . . 3

2a. Sin capuchón. Cola de siete a diez veces el tamaño del tronco, Un ovario esférico y un testículo ovalado ..........

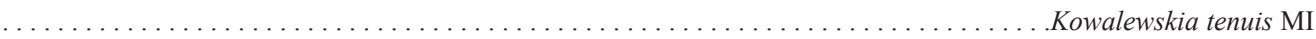

2b. Con capuchón. Cola de cuatro a cinco veces el tamaño del tronco, dos testículos y un ovario

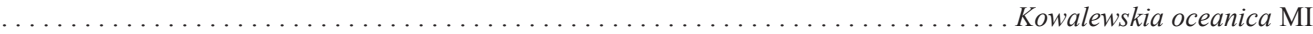

3a. Endostilo recto. Espiráculos a cada lado del recto y ubicados al final de dos canales que conectan la cavidad faríngea

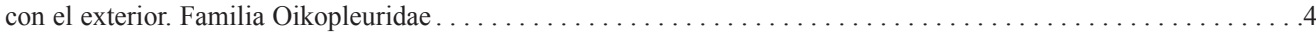

3b. Endostilo de otra forma. Espiráculos conectan la cavidad faríngea directamente con el exterior. . . . . . . . . . .

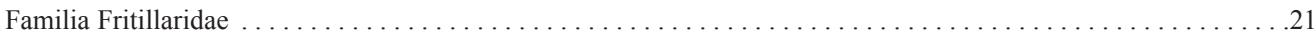

4a. Tronco comprimido dorsoventralmente, boca en posición antero-dorsal. Subfamilia Bathochordaeinae. . . . . . . .5

4b. Tronco ovoide, lateralmente comprimido, boca en posición anterior. Subfamilia Oikopleurinae. . . . . . . . . . .

5a. Boca con un labio inferior. Tracto digestivo orientado lateralmente, espiráculos cortos. Cavidad faríngea del mismo

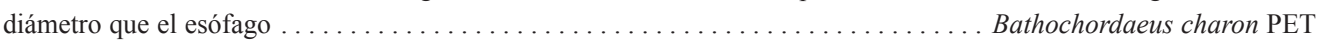

5b. Boca con cuatro labios. Tracto digestivo orientado longitudinalmente, estómago orientado ligeramente hacia la izquierda, intestino ligeramente hacia la derecha. Espiráculos alargados. Género Mesochordaeus . . . . . . . . . . .6

6a. Espiráculos rectos. Parte distal de la cola redondeada . . . . . . . . . . . . . . . Mesochordaeus bahamasi MI

6b. Espiráculos cóncavos hacia la cavidad faríngea. Parte distal de la cola con una muesca marcada

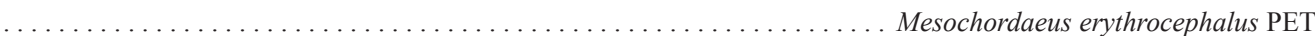

7a. Boca sin labio inferior y rodeada de cilios sensoriales. Supergénero Alabiata $\ldots \ldots \ldots \ldots \ldots \ldots \ldots$

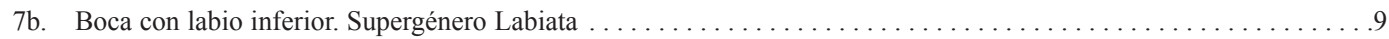

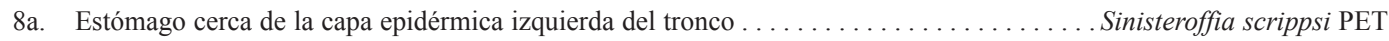

8b. Estómago ubicado en la mitad del tronco y redondo en vista lateral (Figura 6A) .......Pelagopleura verticalis PET

9a. Estómago dividido en dos lóbulos laterales. Género Oikopleura $\ldots \ldots \ldots \ldots \ldots \ldots \ldots \ldots \ldots \ldots \ldots \ldots \ldots$

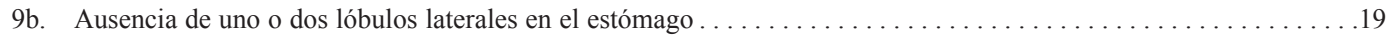

10a. Glándulas bucales y células subcordales presentes (Figura 4E, F). Subgénero Vexillaria . . . . . . . . . . . . 11

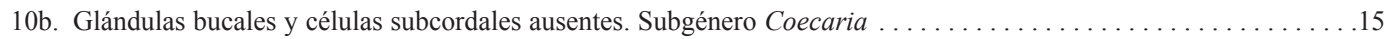

11a. Células subcordales dispuestas en dos filas extendidos por la mayor parte de la cola. Lóbulo izquierdo con un ciego

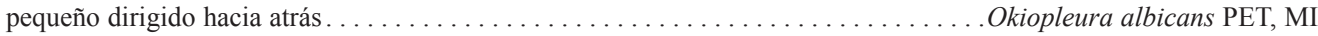

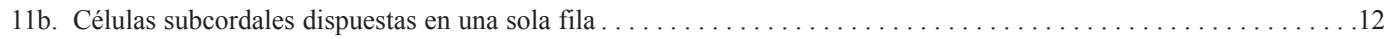

12a. De 4-8 células subcordales globulares, usualmente dispuestas en dos grupos (Figura 4D) .

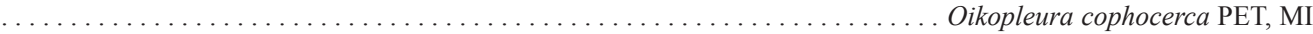

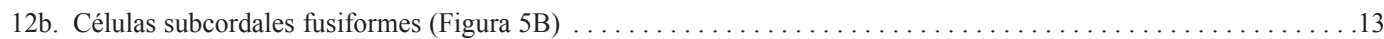

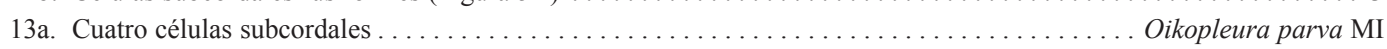

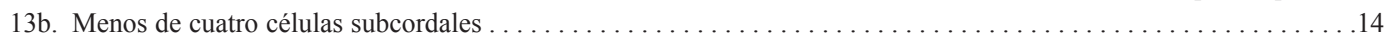

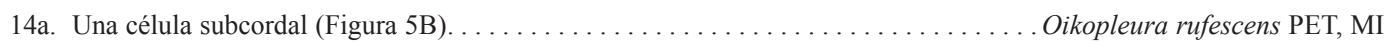

14b. Dos células subcordales (Figura $4 \mathrm{~F}) \ldots \ldots \ldots \ldots \ldots \ldots \ldots \ldots \ldots \ldots \ldots \ldots$. $\ldots \ldots \ldots \ldots \ldots$ dioica $\mathrm{PET}$, MI

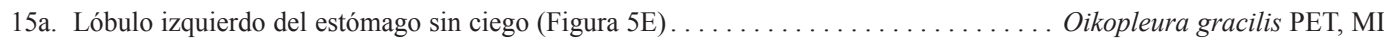

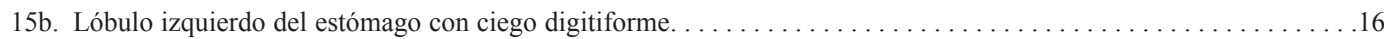

16a. Extremo distal del ciego digestivo separado del lóbulo izquierdo del estómago ..... Oikopleura intermedia PET, MI

16b. Extremo distal del ciego digestivo cerca del lóbulo izquierdo del estómago $\ldots \ldots \ldots \ldots \ldots \ldots \ldots \ldots \ldots$

17a. Ciego digestivo no toca la pared dorsal del tronco, con velo dorsal que cubre la parte media y posterior del tronco

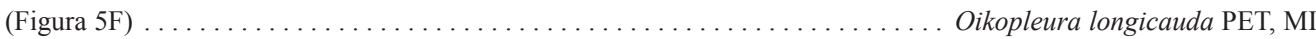

17b. Ciego digestivo se proyecta hasta tocar la pared del tronco, sin velo dorsal. . . . . . . . Oikopleura fusiformis. . 18

18a. Ciego digestivo se proyecta dorsalmente (Figura 5A) ........... Oikopleura fusiformis cornutogastra PET, MI

18b. Ciego digestivo se proyecta primero dorsalmente y luego posteriormente (Figura 5D).

$\ldots \ldots \ldots \ldots \ldots \ldots \ldots \ldots \ldots \ldots \ldots \ldots \ldots \ldots \ldots \ldots \ldots \ldots \ldots$ Oikopleura fusiformis typica PET, MI 
19a. Tracto digestivo con forma de "U" abierto hacia el frente, Lóbulo izquierdo del estómago grande, y emerge de un ducto

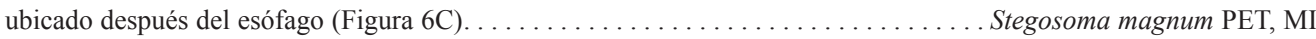

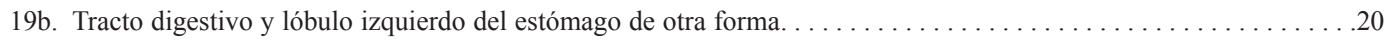

20a. Lóbulo izquierdo del estómago grueso. Estómago orientado verticalmente, esófago se inserta en la parte dorsal del

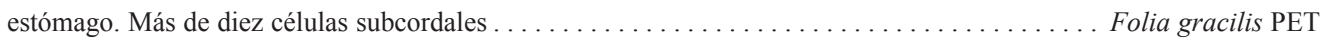

20b. Lóbulo izquierdo del estómago delgado, tubular y dirigido hacia delante. Endostilo separado del ano............

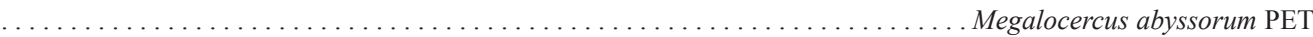

21a. Boca sin labios y rodeada de cilios. Tronco en forma de pera. Cola con una muesca en la parte distal. Endostilo trian-

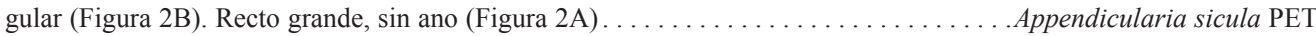

21b. Boca con labios. Tronco aplanado dorsoventralmente (Figura 2C). Endostilo de otra forma, generalmente curvo hacia

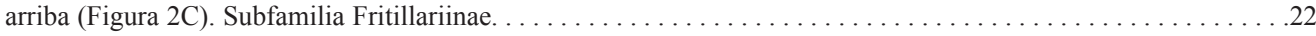

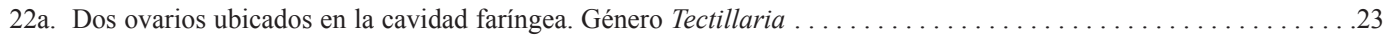

22b. Ovarios ubicados detrás del estómago (Figura 3B). Género Fritillaria $\ldots \ldots \ldots \ldots \ldots \ldots \ldots \ldots \ldots \ldots \ldots \ldots$

23a. Testículos gruesos, cilíndricos y curvos hacia arriba en la parte posterior, limitados a la parte posterior del tronco. Ovarios ubicados a los lados de los espiráculos................... Tectillaria fertilis PET, MI

23b. Testículos delgados, se extienden desde la parte posterior del tronco hacia los ovarios, los cuales están ubicados entre

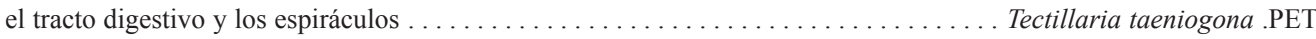

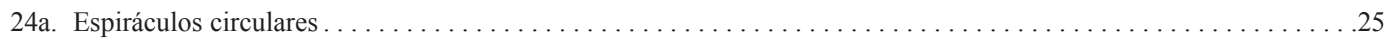

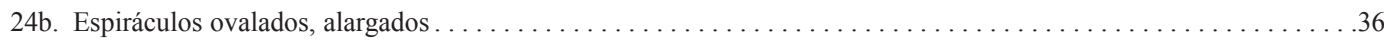

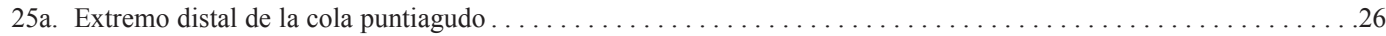

25b. Extremo distal de la cola con una muesca o truncado . . . . . . . . . . . . . . . . . . . . . . . . . . 29

26a. Tronco comprimido dorso-ventralmente y ancho. Testículo en forma de $\mathrm{C}$, en ambos extremos del testículo se encuen-

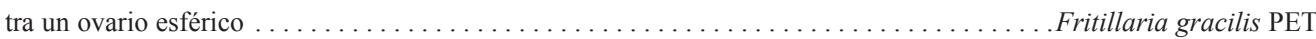

26b. Tronco alargado. Un ovario esférico, posterior a este se encuentra un testículo alargado hacia la parte posterior del

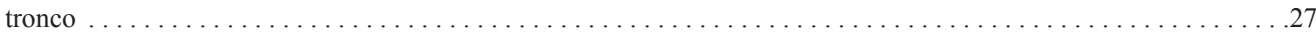

27a. Dos labios con dos lóbulos laterales grandes y uno medio más pequeño (Figura 2E), musculatura de la cola ancha

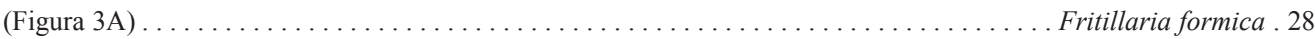

27b. Un labio dorsal alargado y sin lóbulos, musculatura de la cola delgada (Figura 3C) . Fritillaria haplostoma PET, MI

28a. Lóbulo medio del labio superior corto y con dos procesos digitiformes. Testículo triangular cuando está maduro .... Fritillaria formica digitata. PET, MI

28b. Lóbulo medio del labio superior largo y con dos grupos de cilios. Testículo cilíndrico cuando está maduro ......... $\ldots \ldots \ldots \ldots \ldots \ldots \ldots \ldots \ldots \ldots \ldots \ldots \ldots \ldots \ldots \ldots \ldots \ldots \ldots \ldots \ldots \ldots \ldots \ldots \ldots \ldots$ PETillaria formica tuberculata $\mathrm{PE}$

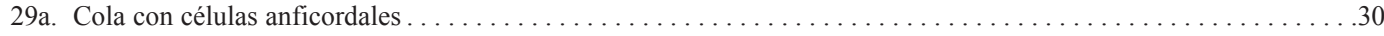

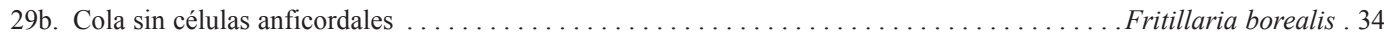

30a. Tronco alargado longitudinalmente y delgado. Dos pares de células anficordales, las cuales son aplanadas y están

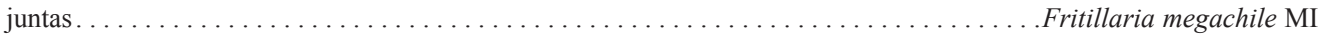

30b. Tronco comprimido dorso-ventralmente, casi rectangular. De uno a dos pares de células anficordales. Con dos proyec-

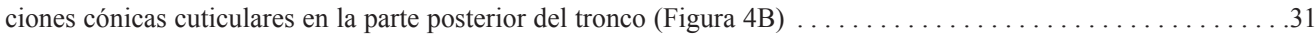

31a. Dos pares de células anficordales globulares con canales marcados (Figura 4A)........Fritillaria pellucida MI . 32

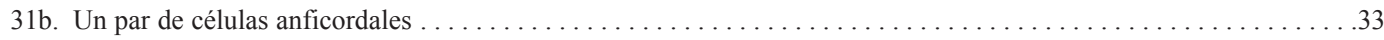

32a. Testículo un lóbulo diferenciado que le da una forma de "r" a la gónada. Proyecciones cuticulares posteriores casi tan

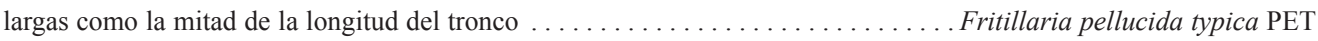

32b. Testículo con dos lóbulos bien diferenciados que le dan una forma de "Y" a la gónada (Figura 3F). Proyecciones cuticulares posteriores mucho más cortas que la mitad de la longitud del tronco. . . . Fritillaria pellucida omani PET

33a. Testículo cilíndrico y en posición posteriror con respecto al ovario de forma esférica (Figura 4B). Células anficordales en forma de urna abierta hacia la parte distal de la cola . . . . . . . . . . . . . Fritillaria tenella PET, MI

33b. Testículo triangular, rodeado laterlamente por el ovario. Esta disposición de las gónadas produce un ensanchamiento de la parte posterior del tronco. Células anficordales planas, grandes y translúcidas.........Fritillaria venusta PET

34a. Testículo con dos lóbulos que le da una forma de $\mathrm{Y} \ldots \ldots \ldots \ldots \ldots \ldots \ldots \ldots \ldots$ Fritillaria borealis sargassi $\mathrm{MI}$

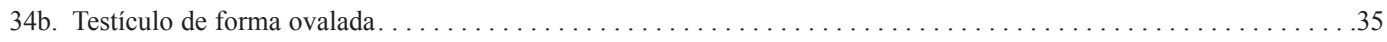

35a. Musculatura de la cola muy angosta, la cual se hace más angosta hacia la parte distal de la cola ..............

Fritillaria borealis typica $\mathrm{PET}, \mathrm{MI}$ 
35b. Musculatura en la mitad proximal de la cola, la cual se adelgaza abruptamente hacia la parte distal . . . . . . . . . . .

Fritillaria borealis intermedia PET, MI

36a. Tres células anficordales de tamaños diferentes a cada lado de la cola (Figura 3E), endostilo cóncavo hacia arriba (Figura 3D) . . .

Fritillaria pacifica PET

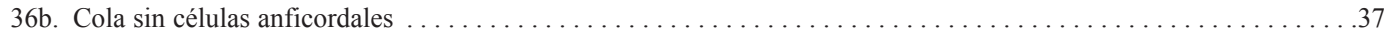

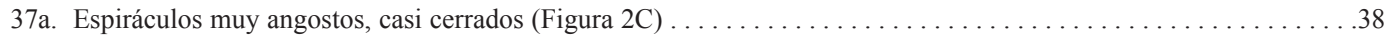

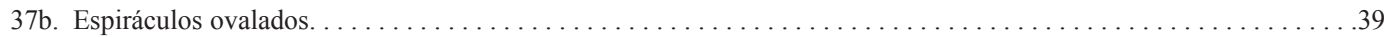

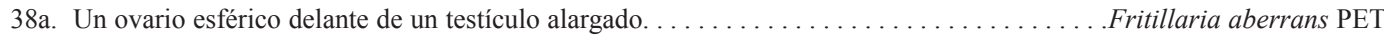

38b. Dos testículos en forma de placas rodeado cada uno de un ovario filiforme. Estómago con un apéndice glandular del

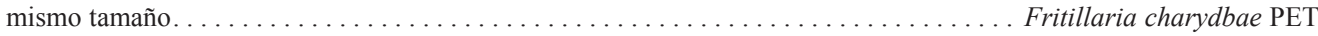

39a. Tronco comprimido dorso-ventralmente, endostilo curvo. Testículo en forma de C, cóncavo hacia la parte anterior del

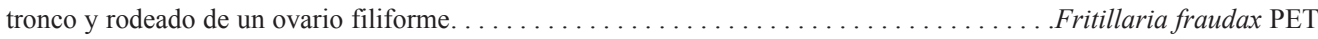

39b. Tronco alargado longitudinalmente, endostilo ligeramente curvo. Testículo ovalado y ovario filiforme ambos alargados longitudinalmente

Fritillaria aequatorialis PET

\section{DISCUSIÓN}

En el Pacífico Costarricense el número de especies de apendicularias reportadas constituye el $41.8 \%$ del total de especies descritas para el Océano Pacífico, donde se conocen 43 especies (Fenaux, Bone, \& Deibel, 1998). Este trabajo incluyo el análisis de muestras recolectadas con redes de 500, 200 y $100 \mu \mathrm{m}$ de apertura de poro, lo que permitió recolectar especies con distintas tallas (Fenaux \& Palazzoli, 1979; Fenaux et al., 1998), incluyendo especies de tamaño pequeño $(\sim 100 \mu \mathrm{m})$ como Appendicularia sicula, Fritillaria charydbae, Fritillaria cf. pacifica, Fritillaria tenella, Fritillaria pellucida f. omani, Oikoipleura fusiformis f. cornutogastra y apendicularias grandes $(\sim 500 \mu \mathrm{m})$ como Pegalopleura verticalis, son nuevos registro para aguas del Pacífico de Costa Rica.

Por otro lado, solo el $6.38 \%$ de las 47 apendicularias reportadas para el Caribe fueron encontradas en este estudio (Fenaux et al., 1998; Castellanos \& Suarez-Morales, 2009). Las tres especies $O$. longicauda, F. haplostoma y Fritillaria formica, son las mismas reportadas para el sistema arrecifal de Cahuita, y se ha determinado que son uno de los grupos más abundantes del holozooplancton (Carrillo, 2012). En la región de Bocas del Toro, Panama, se ha encontrado la especie $O$. fusiformis (obs. pers.) lo cual sugiere que dicha especie también esta presente en el Caribe costarricense.
En este estudio se encontró un total de 18 especies, otros estudios que han recolectado muestras en ambientes epipelagicos de la región han encontrado un número similar de las mismas especies: frente a las costas de California Fenaux \& Dallot (1980) observaron 23 especies de apendicularias; en las costas de Perú, Fenaux (1968) registró 9 especies; y en las costas de Chile, Aravena \& Palma (2002) reportaron 15 especies. Muestras de zooplancton recolectadas a intervalos de profundidad de 100 a 600 m en el Océano Pacifico han encontrado especies como Sinisteroffia scrippsi, Fritillaria taeniogona, Fritillaria aequatorialis, Tectillaria fertilis, Megalocercus abyssorum, Folia gracilis, y Fritillaria aberrans (Tokioka 1957, 1958), las cuales no han sido reportadas en los estudios anteriores. Esto se debe a que la fauna de ambientes epipelágicos $(<200 \mathrm{~m})$ es distinta a la de ambientes meso y batipelágicos (200-3000 m) (Robison, 2004).

La comunidad de apendicularias presente en una region también varía según las distintas provincias biogeográficas de aguas superficiales (Van der Spoel \& Heyman, 1983; Fenaux et al., 1998; Spalding et al., 2012), y por lo tanto se pueden utiizar para determinar la conectividad de distintas regiones. Por ejemplo, las especies $P$. verticalis y $F$. charybdae han sido reportadas en el Pacífico Occidental (Tokioka, 1955), en el Pacífico Oriental (Fenaux \& Dallot, 1980) y en este estudio, lo que sugiere una gran conectividad entre las comunidades 
zooplanctónicas de estas regiones pelágicas. Cuando se analiza la composición de especies de apendicularias a escalas regionales, se ha encontrado que las especies varían según las distintas masas de agua: en la costa de California, las aguas con origen en el Pacifico Central poseen una comunidad distinta a las aguas del Pacifico Ecuatorial.

Este estudio proporciona una guía de identificación para las apendicularias de Costa Rica, lo cual facilitara relacionar la composición de especies de apendicularias a los procesos oceanográficos prevalentes de la región Pacifico y Caribe. Estudios futuros deben caracterizar la variación espacial y temporal de la abundancia de apendicularias como método indirecto para evaluar su relevancia trofica en los ambientes marinos de Costa Rica (Scheinberg, 2004). Estos datos junto con una caracterización detallada de las especies y mediciones de temperatura y salinidad permitirán desarrollar una línea base para determinar los procesos naturales que determinan su distribución (Acuña \& Anadón, 1992), y detectar a futuro las anomalías que puedan surgir conforme los océanos experimentan cambios ecológicos (Richardson, 2008).

\section{AGRADECIMIENTOS}

Agradecemos a Eleazar Ruiz, Antonio Perez-Perez, y la empresa Undersea Hunter por el apoyo durante la recolección de las muestras. Agrademos a Jorge Cortés por permitirnos participar en las giras a Bahía Salinas dentro del Proyecto 808-A5- 037: Comunidades bentónicas y caracterización física y química de Bahía Salinas, Guanacaste. Este proyecto se llevó a cabo gracias al apoyo de la Vicerrectoría de Investigación de la Universidad de Costa Rica a través de los proyectos 808-3A- 062 y 808 B0-060. Agradecemos a dos revisores anonimos por sus comentarios, los cuales mejoraron significativamente la calidad del texto.

\section{RESUMEN}

A pesar de que las apendicularias son consumidores prirmarios relevantes en las comunidades planctónicas, existe poca información detallada sobre las especies presentes en distintas regiones oceánicas. Por lo tanto, las guías regionales de identificación son importantes para desarrollar bases de datos con alta resolución taxonómica. En este trabajo, identificamos las especies de apendicularias presentes en 33 muestras epipelágicas de zooplancton recolectadas en varias localidades dentro de aguas costarricenses. 18 especies de apendicularias fueron identificadas, de las cuales 15 fueron recolectadas en el Pacífico y solo tres fueron recolectadas en el Caribe. Siete especies son registros nuevos para el Pacífico costarricense (Appendicularia sicula, Fritillaria charybdae, $F$. cf. pacifica, $F$. tenella, F. pellucida f. omani, Oikopleura fusiformis f. cornutogastra y Pelagopleura verticalis). Presentamos datos sobre la distribución en Costa Rica de cada especie, junto con una clave dicotómica para todas las especies reportadas previamente para los mares Interamericanos y el Pacífico Tropical Oriental.

Palabras clave: Apendicularias, Costa Rica, clave de identificación, mar Caribe, zooplancton, taxonomía, lista de especies.

\section{REFERENCIAS}

Acuña, J. L., \& Anadón, R. (1992). Appendicularian assemblages in a shelf area and their relationship with temperature. Journal of Plankton Research, 14(9), 1233-1250.

Alldredge, A. L. (1976). Discarded appendicularian houses as source of food, surface habitats, and particulate organic matter in planktonic environments. Limnology and Oceanography, 21(1), 14-23.

Alldredge, A. L. (1981). The impact of appendicularian grazing on natural food concentrations in situ. Limnology and Oceanography, 26(2), 247-257.

Alldredge, A. L. \& Madin, L. P. (1982). Pelagic tunicates. Unique herbivores in the marine plankton. Bioscience, 32, 655-663.

Aravena, G. \& Palma, S. (2002). Taxonomic identification of appendicularians collected in the epipelagic waters off northern Chile (Tunicata, Appendicularia). Revista Chilena de Historia Natural, 75(2), 307-325.

Bedo, A. W., Acuña, J. L., Robins, D. \& Harris, P. H. (1993). Grazing in the micron and submicron size range: the case of Oikopleura dioica. Bulletin of Marine Science, 53(1), 2-14.

Carrillo, A. (2012). Diversidad, abundancia, composición y biomasa del zooplancton de la zona arrecifal del Parque Nacional Cahuita, Limón ¿Cuál es la 
disponibilidad de larvas de invertebrados bénticos 25 años después? (Tesis de Licenciatura). San José, Costa Rica: Universidad de Costa Rica.

Castellanos, I., \& Suarez-Morales, E. (2009). Appendicularia (Urochordata) of the Gulf of Mexico. En D.R Felder \& D.K. Camp (Eds.). Gulf of Mexico- Origins, Waters, and Biota. (Volume I: Biodiversity, pp. 1217 1221). Texas, EEUU: Texas A\&M University Press.

Castellanos, I., Hernández, R., Morales-Ramírez, A., \& Corrales-Ugalde, M. (2012). Apendicularias (Urochordata) y Quetognatos (Chaetognatha) del Parque Nacional Isla del Coco, Costa Rica. Revista de Biología Tropical, 60(3), 243-255.

Costello, M. J., Coll, M., Danovaro, R., Halpin, P., Ojaveer, H., \& Miloslavich, P. (2010). A census of marine biodiversity knowledge, resources, and future challenges. PLOS one, 5 (8), 1-15.

Davoll, P. J. \& Youngbluth, M. J. (1990). Heterotrophic activity on appendicularian (Tunicata: Appendicularia) houses in mesopelagic regions and their potential contribution to particle flux. Deep-Sea Research, $37(2), 285-294$.

Esnal, G. (1996). Thaliacea y Appendicularia. En R. Gasca \& E. Suárez (Eds.). Introducción al Estudio del Zooplancton Marino (pp. 597-630). México. El Colegio de la Frontera Sur (ECOSUR)/CONACYT.

Fenaux, R. (1968). Algunas apendicularias de la costa peruana. Boletin del Instituto del Mar de Perú, 1(9), 536-552.

Fenaux, R. (1993). The classification of Appendicularia: history and current state. (Memoires de L'Institut Oceanographique. No. 17). Mónaco.

Fenaux, R. (1998a). The classification of Appendicularia. En Q. Bone (Ed.). The Biology of Pelagic Tunicates (pp. 295-306). New York, EEUU: Oxford University Press.

Fenaux, R. (1998b). Anatomy and functional morphology of the Appendicularia. En Q. Bone (Ed.). The Biology of Pelagic Tunicates (pp. 25-34). New York, EEUU: Oxford University Press.

Fenaux, R., \& Dallot, S. (1980). Répartition des apendiculaires au large des côtes de Californie. Journal of Plankton Research, 2(2), 145-167.

Fenaux, R. \& Palazzoli, I. (1979). Estimation in situ d'une population d'Oikopleura longicauda (Appendicularia) à l'aide de deux filets de maille différente. Marine Biology, 55(3), 197-200.

Fenaux, R., Bone, Q., \& Deibel, D. (1998). Appendicularian distribution and zoogeography. En Q. Bone (Ed.). The Biology of Pelagic Tunicates (pp. 295306). New York, EEUU: Oxford University Press.

Flood, P. R., \& Deibel, D. (1998). The appendicularian house. The Biology of Pelagic Tunicates (pp
105-124). New York, EEUU: Oxford University Press.

López-Urrutia, A., Irigoien, X., Acuña, J. L., \& Harris, R. (2003). In situ feeding physiology and grazing impact of the appendicularian community in temperate waters. Marine Ecology Progress Series, 252, 125-141.

Madin, L. P., \& Deibel, D. (1998). Feeding and energetic of Thalacea. En Q. Bone (Ed.). The Biology of Pelagic Tunicates (pp. 81-104). New York, EEUU: Oxford University Press.

Richardson, A. J. (2008). In hot water: zooplankton and climate change. ICES Journal of Marine Science, 65, 279-295.

Robison, B. H. (2004). Deep pelagic biology. Journal of Experimental Marine Biology and Ecology, 300, 253-272.

Scheinberg, R. D. (2004). Food web structure and trophic dynamics of a subtropical plankton community, with an emphasis on appendicularians (Tesis de Doctorado). Hawaii, Estados Unidos: Universidad de Hawaii.

Spalding, D., Agostini, V. N., Rice, J., \& Grant, S. (2012). Pelagic provinces of the world: a biogeographic classification of the world's surface pelagic waters. Ocean and Coastal Management, 60,19-30.

Stachowicz J. J., Terwin, J. R., Whitlatch, R. B., \& Osman, R. W. (2002). Linking climate change and biological invasions: ocean warming facilitates nonindigenous species invasions. Proceedings of the National Academy of Sciences, 99(24), 15497-15500.

Tokioka, T. (1951). Pelagic Tunicates and Chaetognaths collected during the cruises to the new Yamato Bank in the Sea of Japan. Publications of the Seto Marine Biological Laboratory, 2(1), 1-25.

Tokioka, T. (1955). General considerations on Japanese appendicularian fauna. Publications of the Seto Marine Biological Laboratory, 4(2-3), 251-261.

Tokioka, T. (1957). Two new appendicularians from the Eastern Pacific, with notes on the morphology of Fritillaria aequatorialis and Tectillaria fertilis. Transactions of the American Microscopical Society, 76(4), 359-365.

Tokioka, T. (1958). Further notes of some appendicularians from the Eastern Pacific. Publications of the Seto Marine Biological Laboratory, 7(1), 1-17.

Tokioka, T., \& Suárez-Caabro, J. (1956). Apendicularias de los mares cubanos. Memorias de la Sociedad Cubana de Historia Natural, 23, 37-95.

Van der Spoel, S., \& Heyman, R. P. (1983). A comparative atlas of zooplankton: biological patterns in the ocean, Berlín, Alemania: Springer. 